\title{
CONDITIONALS AND THEORY CHANGE: REVISIONS, EXPANSIONS, AND ADDITIONS*
}

\begin{abstract}
This paper dwells upon formal models of changes of beliefs, or theories, which are expressed in languages containing a binary conditional connective. After defining the basic concept of a (non-trivial) belief revision model. I present a simple proof of Gärdenfors's (1986) triviality theorem. I claim that on a proper understanding of this theorem we must give up the thesis that consistent revisions ('additions') are to be equated with logical expansions. If negated or 'might' conditionals are interpreted on the basis of 'autoepistemic omniscience', or if autoepistemic modalities (Moore) are admitted, even more severe triviality results ensue. It is argued that additions cannot be philosophically construed as "parasitic' (Levi) on expansions. In conclusion I outline somed logical consequences of the fact that we must not expect 'monotonic' revisions in languages including conditionals.
\end{abstract}

\section{BELIEF REVISION MODELS}

Beliefs and theories are expressed by way of sentences of a certain language. In this paper I shall presuppose a language $L$ that contains the operators of propositional logic $(\sim, \&, \vee, \rightarrow)$ and allows the formulation of conditionals of the form 'If A then B'. As in natural language, conditionals will not be given a special syntactical treatment in advance. In particular I do not exclude negations of conditionals.

Belief revision models (BRMs) are formal models of the dynamics of epistemic states or theories. A belief set, or theory, is a set of sentences of a language $L$ which is closed under a logic (consequence relation, derivability relation) $\vdash$ for $L$. The logic will be supposed to include classical propositional logic, in particular modus ponens, and the deduction theorem is required to hold for $t$.

At first we shall consider only one kind of theory change, viz., so-called 'revisions'. By the revision of a theory $K$ we mean the assimilation of a sentence $A$ into $K$. Theory revisions figure, for example, in processing a new piece of factual information or in following the lines of a hypothetical argument. We can distinguish two cases. If $A$ is consistent with $K$ (under $\vdash$ ), the revision seems to be a matter of routine. But if $A$ is inconsistent with $K$ (under $\vdash$ ), then the affair will become tricky. It is for the sake of such belief-contravening changes 
that BRMs were introduced. The following model for theory change, however, is intended to cover both kinds of revisions. It is important that revisions ought to be economical or minimal, i.e., they should not waste information contained in $K$ without need.

This paper is based on a simplified form of the BRMs initiated by Peter Gärdenfors. A belief revision model, or BRM, is an ordered couple $\langle\mathbf{K}, *\rangle$ such that $\mathbf{K}$ is a set of belief sets and $*: \mathbf{K} \times \operatorname{Sent}(L) \rightarrow \mathbf{K}$ is a function assigning a revision $*(K, A)$ to any belief set $K \in \mathbf{K}$ and any $L$-sentence $A$. We shall abbreviate $*(K, A)$ by $K_{A}^{*}$ in what follows. $K_{A}^{*}$ again is a belief set and is to denote the minimal revision needed to assimilate $A$ into $K$. We presuppose that $*$ is intensional: For all $A$ and $B$ equivalent under $\vdash, K_{A}^{*}=K_{B}^{*}$.

To make sure that BRMs really at least do in part what we want them to do, we shall require that all BRMs satisfy the following criteria of success, of identity and of consistency:

(S) $\quad A \in K_{A}^{*}$.

(I) If $K$ is r-consistent and $A \in K$, then $K_{A}^{*}=K$.

(C) If both $K$ and $A$ are - -consistent, so is $K_{A}^{*}$.

While (S) and (I) appear to be beyond controversy, (C) could be disputed. ${ }^{1}$ If the sentence $A$ in $(\mathrm{C})$ is hopelessly out of question then an epistemic or 'theoretical' breakdown of $K$ into inconsistency might be regarded as possible. Although I do not think that (C) really is too strong, one might restrict this requirement to sentences $A$ that are 'intuitively innocent'. The arguments to come would have to undergo a similar restriction at various places, but, so I believe, they would suffer no loss of their persuasive power. For this reason I will employ the simpler and still plausible requirement $(\mathrm{C})$.

Notice that (S), (I), and (C) are criteria for BRMs. I have omitted the quantificational prefixes $\forall K \in \mathbf{K} \forall A \in \operatorname{Sentences}(L)$ only for the sake of simplicity. They should not pass out of the reader's mind.

We are interested in BRMs that can serve as models of the potential beliefs or theories of human beings (and artificial intelligence systems). The following definitions are motivated by the fact that believers and theorists are not omniscient.

Let $\langle\mathbf{K}, *\rangle$ be a BRM, and $A$ and $B$ be sentences of $L$. A belief set $K \in \mathbf{K}$ is called $A$-ignorant if neither $A$ nor $\sim A$ is in $K$; it is called $A$-B-ignorant if no (non-tautological) truth-functional combination of $A$ and $B$ is in $K$, i.e., (provided that $A$ and $B$ are logically independent), 
if neither $A \vee B$ nor $A \vee \sim B$ nor $\sim A \vee B$ nor $\sim A \vee \sim B$ is in $K$. A $\mathrm{BRM}\langle\mathbf{K}, *\rangle$ is called non-trivial (weakly non-trivial) if there exists a belief set $K$ in $\mathbf{K}$ and $L$-sentences $A$ and $B$ (an $L$-sentence $A$ ) such that $K$ is $A$ - $B$-ignorant ( $A$-ignorant). Clearly, $A$ - $B$-ignorance implies $A$-ignorance, and non-triviality implies weak non-triviality.

I assume that any BRM intuitively should be non-trivial and satisfy (S), (I), and (C). Nevertheless, I shall make explicit where these presuppositions are actually made use of in the following.

\section{GÄRDENFORS'S TRIVIALITY THEOREM}

Perhaps the most important application of BRMs to date has been the analysis of conditionals. Pioneer work was done by Gärdenfors (1978). The key to the interpretation of conditionals is the so-called Ramsey test. It originated in a footnote of Frank P. Ramsey's (1931, p. 247) and can very naturally be expressed as a criterion on BRMs in languages including conditionals:

(R) 'If $A$ then $B$ ' $\in K$ iff $B \in K_{A}^{*}$.

Gärdenfors developed the analysis of conditionals by means of $(R)$ and his concept of BRMs very far. But recently he himself has struck his own approach a heavy blow. In Gärdenfors (1986), he stated the following now notorious triviality theorem: There is no non-trivial BRM that satisfies $(\mathrm{S}),(\mathrm{C}),(\mathrm{R})$, and $(\mathrm{P}) .^{2}$

Here $(\mathrm{P})$ is a prima facie most plausible criterion of preservation: If the sentence $A$ with respect to which the revision of $K$ is to be made is consistent with $K$, then $K_{A}^{*}$ loses nothing of the contents of $K$. More formally,

(P) If $\sim A \notin K$, then $K \subseteq K_{A}^{*}$.

I would like to present a very simple proof for the theorem which is easier to grasp than the proof in Gärdenfors (1986). Its perspicuity may aid us in seeing 'the reasons' for the unexpected rise of triviality. As may easily be verified by the reader, all results gained from the following proof can be carried over to Gärdenfors's original proof.

Let $\langle\mathbf{K}, *\rangle$ be a non-trivial BRM and let $K \in \mathbf{K}$ be $A$ - $B$-ignorant. The gist of the proof is illustrated by Figure 1 . 


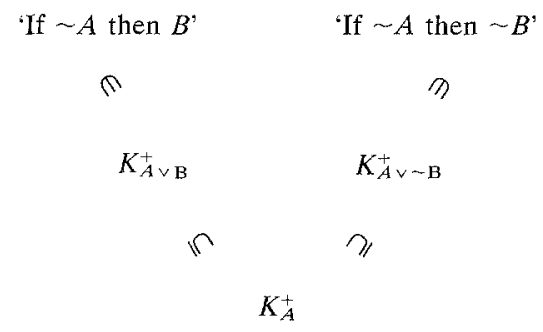

Fig. 1 .

Here $K_{A}^{+}\left(K_{A \vee B}^{+}, K_{A \vee \sim B}^{+}\right.$denotes the expansion of $K$ by $A$ (by $A \vee B$ and $A \vee \sim B$, respectively), as defined by

$(\mathrm{Def}+) K_{A}^{+}$is the set of $\vdash$-consequences of $K \cup\{A\}$, or equivalently, ${ }^{3}$ $K_{A}^{+}={ }_{\operatorname{def}}\{C: A \rightarrow C \in K\}$.

Note that $A \in K_{A}^{+}$and $K \subseteq K_{A}^{+}$.

Let us now exploit the premises of Gärdenfors's triviality theorem and write down the proof in detail.
(1) $A \vee B \in K_{A \vee B}^{+}$
(Deft)
(2) $A \notin K_{A \vee B}^{+}$
$A-B$-ignorance of $K$, (Def+)
(3) $A \vee B \in\left(K_{A \vee B}^{+}\right) *_{A}^{*}$
(1), (2), (P)
(4) $\sim A \in\left(K_{A \vee B}^{+}\right)_{\sim A}^{*}$
(S)
(5) $B \in\left(K_{A \vee B}^{+}\right) *_{A}$
(3), (4), $\left(K_{A \vee B}^{+}\right) *{ }_{A}$ is a belief set
(6) 'If $\sim A$ then $B^{\prime} \in K_{A \vee B}^{+}$
(5), (R)
(7) $K_{A \vee B}^{+} \subseteq K_{A}^{+}$
(Deft)
(8) 'If $\sim A$ then $B$ ' $\in K_{A}^{+}$
(6), (7)
(9) 'If $\sim A$ then $\sim B$ ' $\in K_{A}^{+}$,
analogous to $(1)-(8)$, with $\sim B$ substituted for $B$

The provisional results (8) and (9) to the effect that both 'If $\sim A$ then $B$ ' and 'If $\sim A$ then $\sim B$ ' are in $K_{A}^{+}$conflict with $(\mathrm{R})$ and $(\mathrm{C})$ :
(10) $B, \sim B \in\left(K_{A}^{+}\right)_{A}^{*}$
(11) $\quad\left(K_{A}^{+}\right) *_{A}$ is inconsistent
(12)
$\sim A$ is consistent

$(8),(9),(\mathrm{R})$

(10)

$A-B$-ignorance of $K$ 
$K_{A}^{+}$is consistent

$A-B$-ignorance of $K$

(14) $\quad\left(K_{A}^{+}\right) *_{A}^{*}$ is consistent

(15) Contradiction!

(12), (13), (C)

(11), (14).

Now it looks as though we had managed to show, by using exactly the criteria $(\mathrm{P}),(\mathrm{S}),(\mathrm{R})$, and $(\mathrm{C})$ which are mentioned by Gärdenfors, that only trivial BRMs can meet all four of these criteria.

But have we really been successful? Haven't we overlooked anything? We have. We forgot that in all these criteria we have quantifiers ranging over all $K$ in $\mathbf{K}$. We applied them to expansions quite thoughtlessly. So we tacitly presupposed that if $K$ is in $\mathbf{K}$, then this $\mathbf{K}$ contains the expansions of $K$ as well. Gärdenfors's theorem must read thus:

THEOREM 1 (Gärdenfors). There is no non-trivial BRM $\langle\mathbf{K}, *\rangle$ satisfying $(\mathrm{S}),(\mathrm{C}),(\mathrm{R})$, and $(\mathrm{P})$, that is closed under expansions.

Gärdenfors (1986, p. 85) mentions the closure under expansions only as an incidental technical presupposition. This runs the risk of begging the question in favour of $(P)$, since, as we shall shortly see, the most plausible justification of closure under expansions entails $(\mathrm{P})$. Moreover, at least (S), (C), and (P) appear to be less problematic than this presupposition. We are not facing, as Gärdenfors assumes, a dilemma with two horns labelled $(\mathrm{R})$ and $(\mathrm{P})$, but the theorem sets us an additional task. Why should expansions be in BRMs? The obvious, and perhaps only answer to this question is: Because expansions are a subspecies of revisions. Let us quote from Gärdenfors (1988, p. 54):

The normal application area of a revision process is when the input $A$ contradicts what is already in $K$, i.e., $\sim A \in K$. However, in order to have the revision function defined for all arguments, we can easily extend it to cover the case when $\sim A \notin K$. In this case, the revision is, of course, identified with an expansion.

The suggestion therefore is

(E) If $\sim A \notin K$, then $K_{A}^{*}=K_{A}^{+}$.

Then the closure of BRMs under (consistent) expansions is naturally justified by the very idea and definition of a BRM. Moreover, we can now dispense with $(\mathrm{P})$, which is implied by $(\mathrm{E})$. The theorem can be reformulated thus:

COROLLARY 1. There is no non-trivial BRM satisfying (S), (C), $(\mathrm{R})$, and $(\mathrm{E})$. 
In the face of the dilemma stated in this way, Gärdenfors would presumably plead against $(\mathrm{R})$ and for $(\mathrm{E})$. My aim willd be, however, to show that $(R)$ should be retained and $(E)$ should be given up.

Before turning to a more systematic investigation of the relation between revisions $K_{A}^{*}$ (with $\sim A \notin K$ ) and expansions $K_{A}^{+}$, we note that a certain way out is blocked. The Ramsey test (R) for 'If $A$ then $B$ ' does not require $A$ to be relevant for $B$. In particular, it is a consequence of (I) that 'If $A$ then $B$ ' may, and must, already be accepted when $A$ and $B$ are regarded as true. Rott (1986, pp. 351-52) indicates three variations on $(\mathrm{R})$ as candiates for shunning this intuitive inadequacy:

(R1) 'If $A$ then $B$ ' $\in$ iff $B \in K_{A}^{*} \& B \notin K$.

(R2) 'If $A$ then $B$ ' $\in K$ iff $B \in K_{A}^{*} \& B \notin K_{*}^{*}$.

(R3) 'If $A$ then $B$ ' $\in K$ iff $B \in\left(K_{B}^{-}\right)$.

In (R3), $K_{B}$ denotes the contraction of $K$ with respect to $B$ : The most economical change of $K$ that is necessary to obtain a belief set not including $B$. Like revisions, contractions are required to meet certain criteria. Here we will only need the criteria of success and identity for contractions:

$\left(\mathrm{S}^{-}\right) \quad B \notin K_{B}^{-}$, unless $\vdash B$.

(I $\mathrm{I}^{-} \quad$ If $B \notin K$, then $K_{B}^{-}=K$.

Gärdenfors (1987) showed that (R1)-(R3) do not lead us out of the dilemma. Applying (R1)-(R3) in Figure 1, one realizes that, first, 'If $\sim A$ then $(\sim) B^{\prime}$ remains in $K_{A \vee(\sim) B}^{+}$: for (R1) we need to show that $(\sim) B \notin K$ which is true by supposition; for (R2) we need to show that $(\sim) B \notin\left(K_{A \vee(\sim) B}^{+}\right)_{A}^{*}$ what follows from $(\mathrm{E})$; and owing to $\left(\mathrm{I}^{-}\right)$, there is no change at all for (R3). Secondly, from the fact that both 'If $\sim A$ then $B$ ' and 'If $\sim A$ then $\sim B$ ', we get the contradiction for (R1)-(R3) just as for $(\mathrm{R})$ (for $(\mathrm{R} 3)$ we need $\left(\mathrm{I}^{-}\right)$once more). Thus, we have the following

COROLLARY 2. There is no non-trivial BRM satisfying

$(\mathrm{S}),(\mathrm{C}),(\mathrm{R} 2)$, and $(\mathrm{E})$ or

(c) (S), (C), (R3), (E), and $\left(\mathrm{I}^{-}\right)$.

Hence, installing a relevance condition in the Ramsey test by way of the three variants above does not matter greatly in the present context. We shall keep to the original Ramsey test (R) subsequently. ${ }^{4}$ 


\section{EXPANSIONS AND ADDITIONS}

Having had to put up with the BRMs' closure under expansions in the past section has been unpleasant in some degree. We can gain a better understanding of where additional conditions which are hidden in (E) come in, if we work with consistent revisions, or briefly additions, $K_{A}^{*}$ where $\sim A \notin K$. It is convenient to choose a new notation: in the following, $K_{A}^{\circ}$ denotes the revision of $K$ when it is presupposed, or made sure, that $\sim A$ is not in $K$. (P) and (E), for instance, can then be reformulated as

(P) $K \subseteq K_{A}^{\circ}$.

(E) $K_{A}^{\circ}=K_{A}^{+}$.

Splitting (E) into halves will assist us in our later arguments:

(E1) $K_{A}^{+} \subseteq K_{A}^{\circ}$.

(E2) $K_{A}^{\circ} \subseteq K_{A}^{+}$.

Let us now recast the proof of the triviality theorem. The new idea is represented in Figure 2.

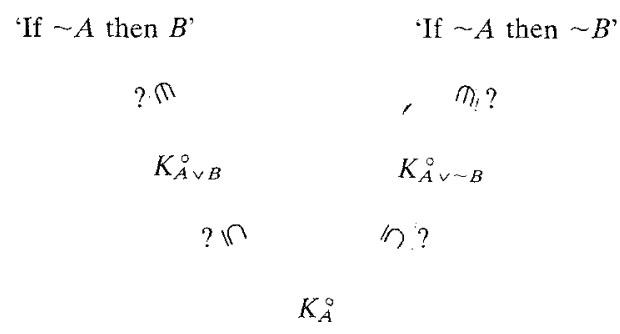

Fig. 2 .

On trying to substitute "o' for ' + ' systematically in steps (1)-(14) in the above proof, we realize that additional assumptions are required at exactly two points, where we referred to $($ Def +$)$. In line (1) we can use (S). But in line (2) we need (E2), and in order to make step (7) we need a condition that I will call monotonicity of additions:

(MA) $K_{A \vee B}^{\circ} \subseteq K_{A}^{\circ}$.

Those who, in contrast to Gärdenfors, are suspicious of condition (P) 
may wish to replace lines (3)-(5) by a new chain of reasoning making use of a condition that can be called conjunctivity of additions:

(CA) $\left(K_{A}^{\circ}\right)_{B}^{\circ}=K_{A \& B}^{\circ}$.

Notice that here it must be checked thrice whether 'o' is allowed to stand in place of '*' ${ }^{5}$ Instead of (3)-(5) above ( ${ }^{\circ}$ ' being substituted for ${ }^{+}$, ) we would come up with

$$
\left(K_{A \vee B}^{\circ}\right)_{\sim A}^{\circ}=K_{A \& B}^{\circ}
$$

(5') $B \in\left(K_{A \vee B}^{\circ}\right) \stackrel{\circ}{\sim} A$ $A-B$-ignorance of $K,(2),(\mathrm{CA})$

Another variation: (CA) would allow us to waive (MA) if we should prefer to employ (P) again. Line (7) above would have to be replaced by the following chain:

$\left(7^{\prime}\right) \quad \sim A \notin K_{A \vee B}^{\circ}$

$\left(7^{\prime \prime}\right) \quad\left(K_{A \vee B}^{\circ}\right)_{A}^{\circ}=K_{A}^{\circ}$

$A-B$-ignorance of $K,(\mathrm{Def}+),(\mathrm{E} 2)$

$\left(7^{\prime \prime \prime}\right) \quad K_{A \vee B}^{\circ} \subseteq K_{A}^{\circ}$

$\left(7^{\prime}\right),(\mathrm{CA})$

$\left(7^{\prime \prime}\right),(\mathrm{P})$

(MA) and (CA) correspond to the monotonicity and conjunctivity of expansions which follow from (Def + ). These conditions are explicitly listed in Peter Gärdenfors's papers $(1986 ; 1987)$ on the triviality results. But what is gained or lost by the various proof variants? In order to get a clear understanding of the mutual dependencies of the conditions proposed, let us collect them all in a little lemma.

\section{LEMMA:}
(a)
$(\mathrm{E}) \Leftrightarrow(\mathrm{E} 1) \&(\mathrm{E} 2)$.
(b)
$(\mathrm{E}) \Rightarrow(\mathrm{MA})$,
$(\mathrm{E}) \Rightarrow(\mathrm{CA})$.
(c)
$(\mathrm{E} 1) \Leftrightarrow(\mathrm{P}) \&\left(\mathrm{~S}^{\circ}\right)$.
(d) $\quad(\mathrm{MA}) \&(\mathrm{I}) \Rightarrow(\mathrm{P})$.
(e) $\quad(\mathrm{CA}) \&(\mathrm{~S}) \&(\mathrm{I}) \Rightarrow(\mathrm{P})$
(f) $\quad(\mathrm{CA}) \&(\mathrm{~S}) \&(\mathrm{I}) \&(\mathrm{E} 2) \Rightarrow(\mathrm{MA})$.

$\left(\mathrm{S}^{\circ}\right)$ in $(\mathrm{C})$ is meant to be the restriction of $(\mathrm{S})$ to consistent revisions:

$\left(\mathrm{S}^{\circ}\right) \quad A \in K_{A}^{\circ}$

Proof of the lemma. (a) Obvious.

(b) As $\vdash$ is a conservative extension of propositional logic and as every belief set $K$ is closed under $\vdash$, we have for all $K$ : if $(A \vee B) \rightarrow C$ 
is in $K$, so is $A \rightarrow C$; and $A \rightarrow(B \rightarrow C)$ is in $K$ iff $(A \& B) \rightarrow C$ is in $K$. Recalling (Def-t), it is now evident that (E) implies (MA) and (CA).

(c) From left to right: for all belief sets $K, A \rightarrow A$ is in $K$, and if $B$ is in $K$, so is $A \rightarrow B$. Hence $K \cup\{A\} \subseteq K_{A}^{+}$, hence, by (E1), also $K \cup\{A\} \subseteq K_{A}^{\circ}$, hence we have $(\mathrm{P})$ and $\left(\mathrm{S}^{\circ}\right)$. From right to left: $(\mathrm{P})$ and $\left(\mathrm{S}^{\circ}\right)$ together imply $K \cup\{A\} \subseteq K_{A}^{\circ}$. Since $K_{A}^{+}$is the smallest set closed under $\vdash$ which contains $K \cup\{A\}$, and since $K_{A}^{\circ}$ as a belief set must be closed under $\vdash$, we have $K_{A}^{+} \subseteq K_{A}^{\circ}$, i.e., (E1).

(d) Take $\sim A$ or $T$ as the $B$ mentioned in (MA).

(e) Let $\sim A \notin K$ and $B \in K$. Then, by (I) and (CA), $K_{A}^{\circ}=\left(K_{B}^{\circ}\right)_{A}^{\circ}=$ $K_{A \& B}^{\circ}$. (The last term denotes an addition, since by supposition $\sim(A \& B) \notin K$. $)$ By $(\mathrm{S})$, we have $B \in K_{A \& B}^{\circ}$, hence $B \in K_{A}^{\circ}$, which proves $(\mathrm{P})$.

(f) Owing to (e), we may use (P). Let $A \notin K$. From (P) and (CA), we get $K_{A \vee B}^{\circ} \subseteq\left(K_{A \vee B}^{\circ}\right){ }_{A}^{\circ}=K_{A}^{\circ}$. Now we are done, if we can show that $\left(K_{A \vee B}^{\circ}\right)_{A}^{\circ}$ really is a twofold addition. To accomplish this we have to verify that $\sim A \notin K_{A \vee B}^{\circ}$ : due to $\sim A \notin K$, we have $\sim A \notin K_{A \vee B}^{+}$, hence, by (E2), also $\sim A \notin K_{A \vee B}^{\circ}$.

QED

It has become evident that more than just $(\mathrm{P})$ is required for the triviality proof: On the one hand, we need (MA) or (CA) which are logically stronger than (P) (if our basic criteria (S) and (I) are presupposed); on the other hand we need (E2). (E) has not to be used to full extent, but it seems that $(\mathrm{E})$ provides the most natural, and possibly the only, motivation of the premises required.

A number of further consequences for the triviality theorem resulting from these observations get clear now. We are not facing the simple alternative of giving up either $(\mathrm{R})$ or $(\mathrm{P})$. A third serious candidate for cancellation has appeared with $(\mathrm{E} 2)$, and $(\mathrm{P})$ has to be strengthened to (CA) or to (MA), both of which also are in want of additional justification. (MA) is a little weaker, since it follows from (CA) given the presence of conditions (S), (I), and (E2), which are indispensable in any case. The most telling form of the triviality result is then cast in the following:

THEOREM 2. There is no non-trivial BRM satisfying (S), (I), (C), (R), (MA), and (E2).

On considering Figure 2 intuitively, one cannot escape the impression that 'If $\sim A$ then $(\sim) B$ ' should be in $K_{A \vee(\sim) B}^{\circ}$, but not in $K_{A}^{\circ}$, if $K$ is $A-B$-ignorant. This is to put the blame on (MA), while (R) and (E2) 
are exonerated in the first instance. The hope, however, that sacrificing (MA) saves (E2) is shortlived. The next section offers separate arguments against both conditions, as well as against (P). Anyhow, (E) certainly does not fit any longer. Expansions are not the right method to 'add' new sentences if the underlying language contains conditionals which are interpreted by the Ramsey test

\section{AUTOEPISTEMIC OMNISCIENCE}

The Ramsey test instructs us to look and see what goes on in $K_{A}^{*}$ : if $B$ is in $K_{A}^{*}$, then - and only then - the conditional 'If $A$ then $B$ ' is in $K$. But what is the matter when $B$ is not in $K_{A}^{*}$ ? I suggest that we should be allowed to put this negative result on record in just the same way as we register the positive one. If $B$ is not in $K$, then, it seems to me, we may - and must - reject the conditional, i.e., accept 'It is not the case that if $A$ then $B$ ', or briefly 'If $A$ then $B$ '. Once the idealizations involved in belief sets and the Ramsey test are agreed to, there is no philosophical reason for repudiating this 'rejectability condition' of conditionals, or acceptability condition of negated conditionals. Ideal epistemic subjects are omniscient with respect to their own belief change. Let us note down the Ramsey-like test for negated conditionals formally:

$$
\text { (R ) } \quad \sim \text { 'If } A \text { then } B^{\prime} \in K \text { iff } B \notin K_{A}^{*} .
$$

In what follows, autoepistemic omniscience ( $A E O)$ is the name of the principle stating that for every belief set $K$ in every BRM $\langle\mathbf{K}, *\rangle$ and every pair of sentences $A$ and $B$, either 'If $A$ then $B$ ' or ' If $A$ then $B^{\prime}$ is in $K .^{6}$ Notice again that this is a principle for BRMs. I think (AEO) is just as plausible as (R), so I rate it as less plausible than the basic criteria $(\mathrm{S}),(\mathrm{I})$, and $(\mathrm{C})$, but as more plausible than $(\mathrm{P}),(\mathrm{E})$, (MA), (E2); "etc.

An ideal subject's omniscience with respect to its own belief change entails its omniscience with respect to its own beliefs. The static trace of the stronger dynamic requirement is obtained by considering the sentences 'If $T$ then $A$ ' and 'If $T$ then $A$ ', where $T$ is an arbitrary tautology or the sentential truth constant. We shall abbreviate these sentences by $\square A$ and $\sim \square A$ respectively. The basic criterion (I) tells us that $\square A$ and $\sim \square A$ are not referring to genuine revisions of the present belief set but to the present belief set itself. Using (AEO) we get the following acceptability conditions: 
(R口) $\square A \in K \quad$ iff $\quad A \in K$.

$(\mathrm{R} \sim \square) \quad \sim \square \in K$ iff $A \notin K$.

Again $(\mathrm{R} \square)$ and( $\mathrm{R} \sim \square)$ are criteria that apply to all $K$ in a $B R M$ $\langle\mathbf{K}, *\rangle$. As they are weaker than the corresponding conditions for (negated) conditionals (if (I) is presupposed), it hardly needs mentioning that there are other ways to discover and justify $(R \square)$ and $(R \sim \square)$ than via the Ramsey test and autoepistemic omniscience. ${ }^{7}$ Seen from this perspective, the following argument is (at least partly) independent from $(\mathrm{R})$. It nevertheless turns against (R)'s 'rivals' in Theorem 2.

Note incidentally that $(\mathrm{R} \sim \square$ ), or already (AEO), excludes the inconsistent set from any BRM $\mathbf{K}$, since it contains all sentences. To avoid a conflict of $(\mathrm{R} \sim \square)$ with $(\mathrm{S}),(\mathrm{S})$ should be restricted in a fashion similar to $\left(\mathrm{S}^{-}\right)$. The new criterion of success then is

(S) $\quad A \in K_{A}^{*}, \quad$ unless $\vdash \sim A$.

Employing ( $\square \square$ ) and $(\mathrm{R} \sim \square)$, we can attack (P) and (E2) one by one.

THEOREM 3. There is no weakly non-trivial BRM satisfying

(a) $\quad(\mathrm{S}),(\mathrm{R} \sim \square)$, and (P) or

(b) (S), (R $\square),(\mathrm{R} \sim \square)$, and (E2).

Proof. Let $K \in \mathbf{K}$ and $A \in \operatorname{Sent}(L)$ be such that $A, \sim A \notin K$.

(a)

(1) $\sim \square \in K \in K \quad A \notin K,(\mathrm{R} \sim \square)$

(2) $\sim \square A \in K_{A}^{\circ} \quad(1), \sim A \notin K$, (P)

(3) $A \notin K_{A}^{\circ}$

(2), ( R $\square)$

(4) $A \in K_{A}^{\circ}$

(S)

(5) Contradiction!

(3), (4).

(b)

(1) $\sim \square A \in K$

$A \notin K,(\mathrm{R} \sim \square)$

(2) $A \in K_{A}^{\circ}$

(S)

(3) $\square A \in K_{A}^{\circ}$

(2), (R $\square)$

(4) $\square A \in K_{A}^{+}$

(3), (E2)

(5) $A \rightarrow \square A \in K$

(4), (Def + )

(6) $\sim \square A \rightarrow \sim A \in K$

(5), propositional logic

(7) $\sim A \in K$

(8) Contradiction!

(1), (6), propositional logic

(7), $\sim A \notin K$.

Now (P) and (E2), and due to the lemma (part (d)) also (MA), i.e., both conditions that were relevant in Theorem 2 have been discredited. 
Moreover, the lemma (part (c)) shows us that both halves of the thesis "consistent revisions are identical with expansions", (E1) and (E2), are untenable if epistemic subjects record their current beliefs by means of sentences of the form $\square A$ and $\sim \square A$.

Let us have a closer look at the proof of part (b) of Theorem 3. The catch in (E2) is that it makes the derivation of (5) possible: if $A \rightarrow \square A$ is in $K$, then $K$ cannot be $A$-ignorant, as is demonstrated in the proof. Thus $A \rightarrow \square A$, or

$$
(T \& A) \rightarrow \text { 'If } T \text { then } A \text { ' }
$$

must not be a sentence scheme derivable in $\vdash$. But it is derivable in the conditional logic of Gärdenfors (1978). This logic is based on the Ramsey test $(\mathrm{R})$ and on the characteristics of Gärdenforsian BRMs. It has

(A) $\quad(A \& B) \rightarrow$ 'If $A$ then $B$ '

as an axiom scheme. Gärdenfors shows that $(\mathrm{A})$ is in every belief set of a BRM, if this BRM satisfies one half of (I), viz.,

$$
\text { If } A \in K \text {, then } K \subseteq K_{A}^{*} \text {. }
$$

We have had no doubts about (I). On the other hand, we have seen that the axiom scheme (A) admits only trivial BRMs, provided that we adopt (R) and (AEO) (or alternatively, $(\mathrm{R} \square)$ and $(\mathrm{R} \sim \square)$ ). What is the reason that we must not take Gärdenfors's conditional logic, which is identical with David Lewis's (1973) 'official' logic VC, as our logic for BRMs?

The crucial point is this. In his proof that - given $(R)-(A)$ is in every belief set $K$, Gärdenfors utilizes the very same premise which he passed over in his triviality proof: the closure of BRMs under expansions. The only justification for this premise would seem to be the thesis that expansions are special cases of revisions, namely additions. But on the strength of the inspection of Figure 2, I have argued that the best way to interpret Gärdenfors's own theorem is to conclude that - given (R) - additions are not to be identified with expansions. Hence the axiom scheme (A) above (as well as other Gärdenforsian axiom schemes) is deprived of its basis. (A) should not be an axiom of conditional logic.

As already mentioned in Section 2, (R) and (I) imply the following: If $A \& B$ is in $K$, then 'If $A$ then $B$ ' is in $K$. This must of course not be confused with $(\mathrm{A})$. On the contrary, we see that it is not warranted 
to infer from the metatheoretical implication 'For all belief sets $K$, if $C$ is in $K$, then $D$ is in $K$ that the corresponding material implication $C \rightarrow D$ is includied in all belief sets.

\section{5. 'MIGHT' CONDITIONALS}

The 'might' conditional 'If $A$ then $\sim B$ might be the case' usually is a more natural expression in the situation described at the beginning of Section 4 (when $B$ is not in $K_{A}^{*}$ ), than the laborious complex sentence 'It is not the case that if $A$ then $B$ '. Instead of $(\mathrm{R} \sim)$, this suggests the following acceptability condition:

$(\mathrm{R} \diamond \rightarrow)^{\text {'If }} A$ then $B$ might be the case' $\in K$ iff $\sim B \notin K_{A}^{*}$.

Accordingly, we define $\diamond A$ by 'If $T$ then $A$ might be the case' and get $(\mathrm{R} \diamond) \quad \diamond A \in K$ iff $\sim A \notin K$.

It is easy to verify that part (a) of Theorem 3 can be proven with $(\mathrm{R} \diamond)$ in just the same way as with $(\mathrm{R} \sim \square$ ). Part (b) requires the (scarcely problematic) additional condition that $\sim \square A$ and $\diamond \sim A$ be mutually exchangeable.

Let us now review a few analyses of 'might' conditionals suggested in the recent literature, and test how they fit in with the definitions above. Lewis (1973) favours a 'not-would-not' reading which can be turned into the following acceptability condition:

(NWN) 'If $A$ then $B$ might be the case' $\in K$ iff

'It is not the case that if $A$ then $B$ ' $\in K$.

Of course, $(\mathrm{NWN})$ is immediately supported by definitions $(\mathrm{R} \diamond \leadsto)$ and $(\mathrm{R} \sim)$. Gärdenfors (1988, pp. 154-156) accepts $(\mathrm{R} \diamond \longrightarrow)$, but not $(\mathrm{NWN})$, because he regards If $A$ then $B$ ' as strictly stronger than 'If $A$ then $B$ might be the case'. The argument he advances in favour of this thesis, however, is a bit weak, and he does not give any comment on the acceptability of negated conditionals. So his rejection of (NWN) cannot wholly convince me.

Lewis (1986, pp. 63-64) contrasts (NWN) with the 'would-bepossible' reading which mirrors the syntactical surface structure of 'might' conditionals best: 
(WBP) 'If $A$ then $B$ might be the case' $\in K$ iff 'If $A$ then $\diamond B$ ' $\in K$.

In our model, (WBP) turns out to be as correct as (NWN): by (R) the right-right side is equivalent to $\diamond B \in K_{A}^{*}$, which in turn is equivalent to $\sim B \notin K_{A}^{*}$ by $(\mathrm{R} \diamond)$. And this latter condition can be changed into the left-hand side via $(\mathrm{R} \prec)$.

Robert Stalnaker's (1981, pp. 98-101) proposal may be termed the 'possibly-would-be' reading of 'might' conditionals and formalized in this way:

(PWB) 'If $A$ then $B$ might be the case' $\in K$ iff $\diamond$ 'If $A$ then $B$ ' $\in K$.

Here, due to (R $\diamond$ ) the right-hand side amounts to $\sim$ 'If $A$ then $B$ ' $\notin K$, which simply means $B \in K_{A}^{*}$ by $(\mathrm{R} \sim)$. On this account, a 'might' conditional could not be distinguished from a pure conditional according to $(\mathrm{R})$. As a consequence of autoepistemic omniscience, the possibility operator $\diamond$. has no effect if placed before a conditional. So from the present point of view Stalnaker's proposal must be repudiated.

\section{WHY ADDITIONS ARE NOT PARASITIC ON EXPANSIONS}

The whole matter would present no problem at all if all belief sets containing 'modalized' sentences, i.e., sentences in which conditional connectives or $\square$ or $\diamond$ occur, were to be constructed in a canonical way from somehow more basic belief sets containing only 'non-modal' sentences. This is the thesis of Isaac Levi (1988):

What is the main feature of the position I am taking about belief revision? It is that all revisions are in the first instance revisions of corpora expressible in nonmodal language L. (p. 70)

$\ldots$ on the view being proposed here, the revisions of corpora expressible in $L^{* *}$ [language with conditionals] are parasitic on the revisions of corpora expressible in $L$ [language of propositional or predicate logic] (p. 66).

Levi excludes conditionals and their relatives from belief sets ('corpora') because in his opinion they don't bear truth values. In Levi (1988), he does not offer reasons why modalized sentences are not capable of being true or false, and I find it hard to understand the pertinent exposition in Levi (1979, pp. 228-31). Recalling the analyses above (to which Levi would principally seem to agree), I guess that this is what 
he has in mind: While the sentences expressible in propositional and predicate logic describe 'the objective world', conditionals (and the like) appear to be only about subjective (changes of) belief. Even if it is not quite clear why this should suffice to refuse conditionals the inclusion in belief sets, it would mean a clear qualitative distinction between non-modal and modal sentences.

Drawn that simply, however, the distinction would rest on a fallacy. One cannot infer from the form of $(R),(R \square),(R \diamond)$ etc. that conditionals are subjective, just as one cannot gather from

$$
A \& B \in K \text { iff } A \in K \text { and } B \in K
$$

that conjunctions are about belief sets. If conditionals really were just about epistemic states, one could not be mistaken about conditionals. This is a consequence of autoepistemic omniscience. But consider the following example:

If Gorbachev had died shortly after his assumption of power, the world's peace would be in greater danger now than it actually is.

Let us take it for granted that this sentence is accepted in our current 'knowledge base' $K$. It is a counterfactual conditional since both the antecedent, $A$, and the consequent, $C$, are presupposed to be false. But if we get the information, from a most reliable source, that Gorbachev really died shortly after his assumption of power, we will revise our beliefs in an entirely different manner than under the hypothetical assumption of $A$. Since $C$ cannot be true in the real world, the conditional must have really been false. It turns out, or is just gathered, that the Soviet Union has secretly put a double in the place of Gorbachev, and that this man has done his job exceedingly well. Anyway, we shall give up the conditional 'If $A$ then $C$ ' in the successive belief set $K^{\prime}$, since $\sim C$, being a good candidate for an analytical truth, resists any rational belief change. ${ }^{8}$

What was the reason for the error we committed by accepting the conditional 'If $A$ then $C$ ' in $K$ ? On the one hand, we were wrong about the constitution of the real world: the world simply has not been such that the unrestricted nuclear arms race continued after (or because of) Gorbachev's untimely death. In $K$, the hypothetical supposition $A$ has reckoned with a world in which $C$ is true. This is why we did accept 'If $A$ then $C$ '. But it was already obvious in $K$ that on being informed 
that Gorbachev really died so soon, the revision would be performed in a completely different fashion: such factual information $A$ would at once make it evident that the conditional accepted in $K$ was erroneous. This example illustrates that conditionals have acceptability conditions referring to hypothetical revisions, but that they are nevertheless means of formulating statements about the real world. Hypothetical suppositions apparently induce revisions that are very different from the revisions caused by new information. ${ }^{9}$ This fundamental distinction to be made between the belief set $K_{A}^{*}$ mentioned in the Ramsey test $(\mathrm{R})$ and the belief set $K^{\prime}$ described in the example above also blocks Gärdenfors's (1988, p. 166) argument against the Ramsey test.

The dynamics of our beliefs, I conclude, mirror reality just as beliefs do. There is no reason why we should not be allowed to clothe these dynamics in conditionals by means of $(R)$ and put them into belief sets. Conditionals are not distinguished syntactically from other complex sentences to which we assign truth values without hesitation. Semantically, they are closely related to disposition predicates, natural laws, and causal expressions which all appear to represent objective features of the world. Levi (1988, pp. 75-78) discriminates truth value bearing pseudo-conditionals that properly express dispositions from genuine conditionals construable according to the belief revision model. But he does not give us ways and means how to recognize and systematically draw the distinction. ${ }^{10}$ Therefore, his hierarchy of first-rate sentences (of propositional and predicate logic) and modalized second-rate sentences, the latter being 'parasitic on' the acceptance of the former, is not convincing.

All the same, let us now assume that a separation of 'objective' and epistemically modalized sentences is possible and necessary. Then we can advance a second strategy against Levi's simplifying view by consulting the arguments of 'non-monotonic' or 'autoepistemic' logics from AI research (see Moore, 1985 and Konolige, 1988). In everyday life we have to make decisions and take actions on the basis of incomplete knowledge. Often we do not know explicitly whether $A$ or $\sim A$ is true, but in countless cases we still add $A$ (and not $\sim A$ ) to our stock of beliefs in order to be able to decide and do anything at all. For example, I do believe that the man I am talking to is not mad, that the car I have just driven is still roadworthy, that the little child's parents are alive, that Tweety, the bird, can fly, and so on. Unless proven otherwise, we take it for granted that these propositions do state the facts, 
and we file these sentences (and not their negations) into our data bases, since they form a considerable part of the basis on which we must orientate ourselves and perform actions.

This kind of sentence $A$ which may be reckoned true 'pending proof to the contrary" or 'under normal circumstances' can be inserted into belief sets as 'default knowledge' by means of 'axioms' of the form $\diamond A \rightarrow A$ (which naturally are not to be read as axiom schemes now). In consistent stable theories of autoepistemic logic (Moore, 1985), the operators $\square$ and $\diamond$ conform exactly to the rules $(R \square)$ and $(R \diamond)$. For any $A$ belonging to the scope of default knowledge, there can plainly be no $A$-ignorance: If you do not explicitly know that $\sim A$, then you will accept $\diamond A$ and the axiom $\diamond A \rightarrow A$ will yield the standard belief that $A$.

In inversion of Levi's dictum, it might here be said that many nondalized sentences $A$ are 'parasitic on' the modalized sentences $\diamond A$. This approach generates interesting philosophical and technical problems of its own, ${ }^{11}$ but it cannot be denied that autoepistemic logic accounts for important aspects of ordinary life reasoning, and it has proven successful, to some degree, at applications in computers. It shows, I believe, that there is no one-way road from 'objective' sentences to epistemically modalized ones.

\section{CONCLUSION}

Gärdenfors's attack on the combination of the Ramsey test (R) with the preservation criterion (P) has not been a full success. The Ramsey test is incompatible with the thesis that consistent revisions may be identified with expansions. To put it more accurately, we are able to demonstrate that $(R)$ clashes with the conjunction of (MA) and (E2). We could not, and neither could Gärdenfors, show a direct effect of (R) upon (P).

While Gärdenfors pleads for $(\mathrm{P})$ and against $(\mathrm{R})$, I have been voting for (R) and against (E). If, after this paper's discussion, one had to decide, what in the whole matter is inadequate, the scapegoat would be spotted as (MA). An unprejudiced look at Figure 2 will urge the conclusion that 'If $\sim A$ then $B$ ' ought to be in $K_{A \vee B}^{\circ}$ but not in $K_{A}^{\circ}$. The triviality proofs of Sections 2 and 3 , therefore, cannot be directed against the Ramsey test.

The problem whether to give up (MA) or (E2) resolves itself when 
autoepistemic omniscience is recognized. Let me once again stress that (AEO) is not an unduly strong requirement in the context of the epistemological model on which this essay is based: idealizations already abound in the very notion of a BRM. On accepting (AEO), however, it becomes evident that both (E2) and (P) - and thus a fortiori (MA) - have intolerable consequences. This was shown by means of negated conditionals in Section 4. If one concentrates on 'might' conditionals (Section 5), the trivialization of (MA) presents no difficulties, while that of (E2) requires as an additional premise that negated and 'might' conditionals, or rather $\sim \square A$ and $\diamond \sim A$, be equivalent. Gärdenfors denies this, but only at the expense of a complete and probably irremediable renunciation of the analysis of negated conditionals.

This renunciation, this gap, is also essential for retaining the Gärdenforsian program for analyzing conditionals by means of the Ramsey test. In Gärdenfors (1978) and in Gärdenfors (1988, ch. 7) the identity of additions and expansions is circumvented solely by Gärdenfors's reluctance to infer $\sim$ 'If $A$ then $B$ ' $\in K$ from $B \notin K_{A}^{*} .{ }^{12}$ In spite of the immunity thus gained, Gärdenfors opts for giving up the Ramsey test in the face of his triviality theorem. This does not seem sufficiently motivated to me. On the other hand, the abandonment of (E) which I have been advocating in this essay also amounts to a decisive blow to the program of Gärdenfors (and that of Rott, 1986). In either case, Gärdenfors's epistemic reconstruction of Lewis's (1973) logic VC for counterfactuals is staggered. At the end of Section 4 we have seen that $\mathrm{VC}$ with its axiom scheme $(A \& B) \rightarrow$ 'If $A$ then $B$ ' cannot be adequate if we adopt autoepistemic omniscience.

It is obvious that $(E)$ is no good if taken together with $(R \sim),(R \sim \square)$, $(\mathrm{R} \diamond \rightarrow)$, or $(\mathrm{R} \diamond)$. As is seen immediately, expansions cannot be included in (weakly) non-trivial BRMs satisfying, say, $(\mathrm{R} \diamond)$ : Let $K$ be an $A$-ignorant belief set; as $A$ is not in $K$, we have $\diamond \sim A$ in $K$ and also in $K_{A}^{+}$; but $A$ is in $K_{A}^{+}$, too, hence $K_{A}^{+}$does not conform to (R $\left.\diamond\right)$, hence $K_{A}^{+}$is not in the BRM. No " $A$-successful" superset of an $A$ ignorant $K$ remains within the range of validity of $(\mathrm{R} \diamond)$. In fact, there cannot be two belief sets in a BRM satisfying ( $\mathrm{R} \diamond)$ that are ordered by proper inclusion: for all $A$ in $K-K^{\prime}$, we have $\diamond \sim A$ in $K^{\prime}-K$.

This grave diagnosis with regard to negated or 'might' conditionals on the one hand, and on the other the intuitive inadequacy of (MA) in Figure 2, arouse the suspicion that there is no monotonic belief change in BRMs based on languages including pure conditionals only. 
Renaming the belief set $K_{A \vee B}^{\circ}$ of Figure 2 in $K^{\prime}$, it is not implausible to suppose that $K_{A}^{\prime \circ}$ is identical with $K_{A}^{\circ}$. Since $K_{A \vee B}^{\circ} \subseteq K_{A}^{\circ}$ is dubious, it then is equally dubious whether $K^{\prime} \subseteq K_{A}^{\prime \circ}$, i.e., the preservation condition (P) applied to $K^{\prime}$, can be met. In Figure 2, 'If $\sim A$ then $B$ ' is thought to be in $K^{\prime}-K_{A}^{\prime \circ}$. Save for very special cases, we always may expect to find conditionals of the form 'If $\sim A$ then . . ' in $K-K_{A}$ for all belief sets $K$ and all sentences $A$. The greater ignorance of $K$ gives way to possibilities which are lost in the more cognizant belief set $K_{A}^{\circ}{ }^{13}$ One can speculate whether there are arguments in support of the claim that no two belief sets in a BRM satisfying ( $R$ ) can be ordered by proper inclusion. But I do not have formal results to this effect.

Starting out from the assumption that in languages including (possibly negated, or 'might') conditionals there cannot be two different belief sets $K$ and $K^{\prime}$ with $K \subseteq K^{\prime}$, it is plain that the monotonicity condition

$$
\text { If } K \subseteq K^{\prime} \text {, then } K_{A}^{*} \subseteq K_{A}^{\prime *}
$$

is entirely innocuous. Gärdenfors (1986) has put (M) in the center of his discussion of the triviality theorem. He notes that $(M)$ immediately follows from (R): Let $B$ be in $K_{A}^{*}$; then we have 'If $A$ then $B$ ' in $K$ by (R), hence, by the antecedent of (M), this latter sentence also is in $K^{\prime}$, hence $B$ is in $K_{A}^{\prime *}$, by $(\mathrm{R})$ again. It may well be that Gärdenfors (1986, pp. 86-87; 1988, pp. 159-160) is basically right in his arguments against $(\mathrm{M})$, but that $(\mathrm{M})$ nevertheless is true - but only trivially so, owing to the nonexistence of proper inclusions between belief sets. Reproaching the Ramsey test for implying $(\mathrm{M})$ does not seem a promising strategy to me. ${ }^{14}$

Another consequence of the theses and results presented above is that it does not make good sense any more to speak of 'expansions' and 'contractions'. ${ }^{15}$ If our object language includes $\sim \square$ or $\diamond$ with acceptability conditions $(\mathrm{R} \sim \square)$ and $(\mathrm{R} \diamond)$, then genuine expansions and contractions simply do not exist. The only kinds of belief or theory change are revisions. Accordingly, it would be less misleading to characterize alleged expansions and contractions as revisions from the start: Instead of $K_{A}^{\circ}$, which essentially is identical with $K_{A}^{+}$in Gärdenfors, ${ }^{16}$ it may be better to write $K_{\square}^{*}$, and instead of $K_{A}^{-}$better $K_{A}^{*}$ or $K_{\diamond}^{*} \sim A \cdot{ }^{17}$ (It is easy to verify that (S) and (I), together with (R $\square$ ), $R \sim \square$, and $(R \diamond)$ respectively, guarantee success and identity of additions and 'contractions' thus defined.) These notations make it obvi- 
ous that every change of belief is, in a sense, at variance with the original beliefs: an addition contradicts an originally admitted possibility, a 'contraction' contradicts an originally endorsed necessity (impossibility). I hope to have made it sufficiently plausible in Section 6 that these contradictions are not artificially grafted on what is basically simple, but are deeply intertwined in the complex web of our beliefs.

Lastly, a logical phenomenon remains irritating. If $\sim A$ is not in $K$, then we would intuitively like to equate $K_{A}^{\circ}$ with the set of consequences of $K$ and $A$ taken together. This, we have seen, is made impossible by, e.g., $(\mathrm{R} \diamond)$. But there is a solution which serves our needs quite well. The idea is that we must desist from understanding a belief set as one single block of beliefs, and decompose it into a body of 'explicit knowledge' $K_{e}$ and a body of 'implicit knowledge' $K_{i}=K-K_{e}$. The entire belief set $K$ is derived from $K_{e}$ with the help of (R $\left.\square\right),(\mathrm{R} \sim \square)$, and $(\mathrm{R} \diamond)$ and some underlying logic $\vdash$. We call $K$ thus constructed the closure of $K_{e}$, in symbols: $K=\mathrm{Cl}\left(K_{e}\right)$. In Section $6 \mathrm{I}$ have argued that as a rule $K_{e}$ will be definitely not simply the non-modal part of $K$, but will contain a lot of 'modal' information.

What have we gained by the decomposition? The standard procedure is to identify $K_{A}^{\circ}$ with the set of r-consequences of $K \cup\{A\}$. Regardless which logic $\vdash$ we use, it will always yield a superset of $K$ and thus violate $(\mathrm{R} \diamond)$. But if we take $\mathrm{Cl}\left(K_{e} \cup\{A\}\right)$ as $K_{A}^{\circ}$, we would of necessity only get a superset of $K$, if the closure operation were monotonic, i.e., if it satisfied

$$
\text { If } K_{e} \subseteq K_{e}^{\prime}, \quad \text { then } \mathrm{Cl}\left(K_{e}\right) \subseteq \mathrm{Cl}\left(K_{e}^{\prime}\right) .
$$

But $\mathrm{Cl}$ is non-monotonic. More generally, research in artificial intelli-

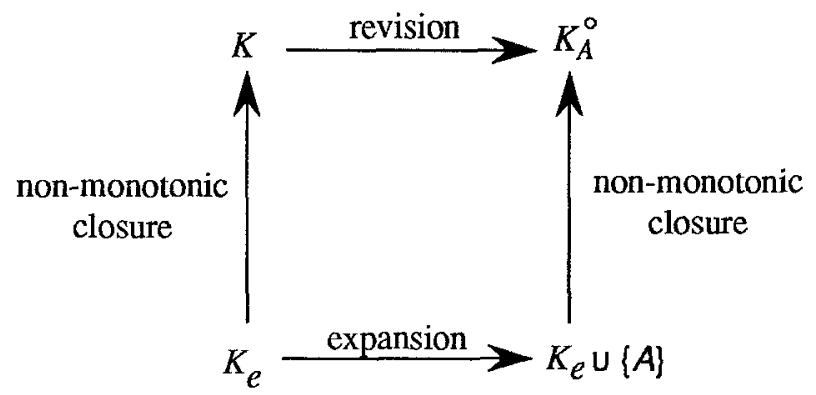

Fig. 3. 
gence indicates that the logic used in everyday life reasoning is nonmonotonic. On decomposing belief sets in the way sketched above and employing a kind of non-monotonic logic, it is finally possible to keep the idea of obtaining consistent revisions (additions) by forming the deductive closure of the old explicit beliefs plus the new sentence to be accepted. The mechanism is illustrated in Figure 3. At least some of the intricacies spotted in the course of this paper appear to be solvable with the help of this construction.

\section{NOTES}

* I wish to thank Peter Gärdenfors for a number of helpful comments, André Fuhrmann and Wolfgang Spohn for extensive discussion of parts of this paper, and Winfred Klink for kindly checking my English.

1 Actually, (C) is raissing in many papers of Gärdenfors. In his epistemological development of Lewis's (1973) system VC (Gärdenfors 1978; 1988, chap. 7) for example, (C) is ignored. This is not surprising, because Lewis allows conditionals to be 'vacuously' true even if their antecedents are noncontradictory, which would be excluded by (C).

${ }^{2}$ Gärdenfors uses a slightly different concept of 'non-trivial BRM'.

${ }^{3}$ Because $t$ includes modus ponens as a (perhaps derived) rule of inference and satisfies the deduction theorem.

${ }^{4}$ Rott (1986) suggested that the principal acceptability condition of conditionals should be a combination of (R2) and (R3), viz.,

$$
\text { 'If } A \text { then } B^{\prime} \in K \text { iff } B \in\left(K_{B}^{-}\right) \stackrel{*}{*} B \notin\left(K_{B}^{-}\right)_{{ }_{A}}^{*} \text {. }
$$

On presupposing $\left(\mathrm{S}^{-}\right),(\mathrm{I})$ and (E) (in fact one only needs the half called (E2) below), however, it is easy to see that (R4) is equivalent to (R4) is equivalent to (R3). Plainly, the right-hand side of (R4) implies the right-hand side of (R3). Conversely, we have to show that $B \notin\left(K_{B}^{-}\right) *_{A}^{*}$ follows from $B \in\left(K_{B}^{-}\right)$. Suppose for reductio that $B$ is in both $\left(K_{B}^{-}\right){ }_{A}^{*}$ and $\left(K_{B}^{-}\right) *_{A}^{*}$. Then (I) implies that neither $A$ nor $\sim A$ can be in $K_{B}^{-}$; otherwise (S $\mathrm{S}^{-}$) would be violated. Using (E), then, the supposition says that both $A \rightarrow B$ and $\sim A \rightarrow B$ are in $K_{\vec{B}}^{-}$, but now, by propositional logic, $B$ is in the belief set $K_{\vec{B}}^{-}$as well, in contradiction to $(\mathrm{S}-)$.

${ }^{5}$ Conjunctivity is definitely out of place as a general requirement for all revisions. See Rott (1988).

${ }^{6}$ Be careful not to confuse autoepistemic omniscience with Stalnaker's (1981) 'Conditional Excluded Middle', i.e., with the axiom scheme 'If $A$ then $B^{\prime} \vee$ 'If $A$ then $\sim B$ '.

${ }^{7}$ The same comment applies to $(R\langle\rangle)$ below. Of particular relevance are Levi's (1979; 1988) 'serious possibility', Moore's (1985) 'autoepistemic logic', and Fuhrmann's (1989) 'reflective modalities'.

${ }^{8}$ Examples that can be utilized in a similar manner are found in Ramsey (1931, p. 249), Mackie (1962, p. 71), Adams (1970, pp. 90), and Stalnaker (1984, pp. 105-106). Presumably there is a close connection between the dichotomies 'new information $v s$. hypothetical supposition' and 'indicative vs. subjunctive conditionals'. 
${ }^{9}$ Both kinds of revision, though, seem to satisfy the same sets of rationality criteria à la Gärdenfors. The difference probably lies in divergent relations of 'epistemic entrenchment' (cf. Gärdenfors 1988, chap. 4) which underlie these revision variants.

${ }^{10}$ There are still more kinds of conditionals in Levi. If for example the antecedent is of the form $\langle>A$, then, according to Levi (1988, pp. 70-72), the conditional must be analyzed by considering the contraction $K_{\sim_{A}}^{-}$. Levi offers no unified analysis of conditionals which could be compared to (R). He thus violates the sound methodological hypothesis that pace Dudman $(1984 ; 1986)$ - different messages be encoded differently. The principal uses of 'if' should be covered by one single explication; 'might', grammatical moods and tenses in conditionals should be accounted for in such a fashion that the principle of compositionality is obeyed. In my opinion, Dudman's work does not conclusively establish that this aim is unattainable.

${ }^{11}$ For instance, there generally is, for a given 'axiom set' $K_{0}$ (closed under first-order consequence), no unique superset $K$ which is first-order closed and satisfies ( $\mathrm{R}(\rangle)$ ). Example: (the first-order closure of) $K_{0}=\{\langle\rangle A \rightarrow \sim B,\langle\rangle B \rightarrow \sim A\}$. If $K_{0}$ is expressible in first-order language, however, existence and uniqueness of $K$ are guaranteed. See Moore (1985) and Konolige (1988).

12 The crucial point is Gärdenfors's condition

$$
\text { If } \sim \text { 'If } A \text { then } \sim B^{\prime} \in K \text {, then }\left(K_{A}^{*}\right)_{B}^{+} \subseteq K_{A \& B}^{*} \text {. }
$$

Using ( $\mathrm{R} \sim$ ) and (I) and substituting $T$ for $A$, we could at once obtain (E) from this condition and Gärdenfors's further condition $K_{A}^{*} \& B \subseteq\left(K_{A}^{*}\right)_{B}^{+}$. Without $(\mathrm{R} \sim)$, this fault does not arise, but then the condition quoted cannot virtually be understood. The critical question is: Under which circumstances is the negated conditional $\sim$ 'If $A$ then $\sim B^{\prime}$ to be accepted in $K$ ?

The analogous problem concerning $(\mathrm{P})$ instead of $(\mathrm{E})$ has already been pointed out by Isaac Levi (1988, pp. 68 and 80).

${ }^{13}$ Unless we are facing the very special case that a 'memory' is built in $K_{A}^{\circ}$ by means of a relation of epistemic entrenchment which dictates that on hypothetically assuming $\sim A$, we should move from $K_{A}^{\circ}$ via $K$ to $K_{-A}^{\circ}$. In general, however, such a memory evaluating the most recent information as lowest does not seem desirable.

${ }_{14}$ To be sure, Gärdenfors has more arguments against the Ramsey test.

${ }^{15}$ My choice of the label 'addition', I admit, is somewhat unhappy, too. It can only be justified by my failure to find a more appropriate name.

${ }^{16}$ But see footnote 12 and the pertaining text.

17 In his arguments against Levi, Fuhrmann $(1989$, p. 14) advances a converse proposal. He defines revisions as special contractions by means of $K_{A}^{*}=K \bar{\sim} \sqsupset A$.

\section{REFERENCES}

Adams, E. W.: 1970, 'Subjunctive and Indicative Conditionals', Foundations of Language 6, 89-94.

Dudman, V. H.: 1984, 'Conditional Interpretations of if-Sentences', Australian Journal of Linguistics 4, 143-204.

Dudman, V. H.: 1986, 'Antecedents and Consequents', Theoria 52, 168-99.

Fuhrmann, A.: 1989, 'Reflective Modalities and Theory Change', Synthese 81, 115-34. 
Gärdenfors, P.: 1978, 'Conditionals and Changes of Belief' in I. Niiniluoto and R. Tuomela (eds.), The Logic and Epistemology of Scientific Change, Acta Philosophica Fennica 30, 381-404.

Gärdenfors, P.: 1986, 'Belief Revisions and the Ramsey Test for Conditionals', Philosophical Review 95, 81-93.

Gärdenfors, P.: 1987, 'Variations on the Ramsey Test: More Triviality Results', Studia Logica 46, 319-25.

Gärdenfors, P.: 1988, Knowledge in Flux: Modeling the Dynamics of Epistemic States, MIT Press, Cambridge, Mass.

Konolige, K.: 1988, 'On the Relation Between Default and Autoepistemic Logic', Artificial Intelligence $\mathbf{3 5}, 343-82$.

Levi, I.: 1979, 'Serious Possibility', in E. Saarinen, R. Hilpinen, I. Niiniluoto, and M. P. Hintikka (eds.), Essays in Honour of Jaakko Hintikka, Reidel, Dordrecht, pp. 219-36.

Levi, I.: 1988, 'Iteration of Conditionals and the Ramsey Test', Synthese 76, 49-81.

Lewis, D.: 1973, Counterfactuals, Blackwell, Oxford.

Lewis, D.: 1986, "Postscripts to "Counterfactual Dependence and Time's Arrow" Philosophical Papers, vol. II, by D. Lewis, Oxford University Press,Oxford, 52-66.

Mackie, J. L.: 1962, 'Counterfactuals and Causal Laws', in R. J. Butler (ed.), Analytical Philosophy, Blackwell, Oxford, pp. 66-80.

Moore, R. C.: 1985, 'Semantical Considerations on Non-monotonic Logic', Artificial Intelligence 25, 75-94.

Ramsey, F. P.: 1931, The Foundations of Mathematics and Other Essays, Kegan Paul, London.

Rott, H.: 1986, 'Ifs, Though, and Because', Erkenntnis 25, 345-70.

Rott, H.: 1988: 'Do Iterated Conditionals Yield a New Argument Against the Ramsey Test for Conditionals?', in preparation.

Stalnaker, R. C.: 1981, 'A Defense of Conditional Excluded Middle', in W. L. Harper, R. C. Stalnaker, and G. Pearce (eds.), Ifs: Conditionals, Belief, Decision, Chance and Time, Reidel, Dordrecht, pp. 87-104.

Stalnaker, R. C.: 1984, Inquiry, Bradford Books, Cambridge.

Seminar für Philosophie, Logik und Wissenschaftstheorie

Ludwig-Maximilians-Universität München

Ludwigstraße 31

D-8000 München 22

West Germany 